\title{
Images - A rare case of Brunn's cyst causing obstructive lower urinary tract symptoms in a young male
}

Runhan Ren; Ryan McLarty; Phil Bach

Division of Urology, University of Alberta, Edmonton, AB, Canada

Cite as: Can Urol Assoc J 2019 November 29; Epub ahead of print. http://dx.doi.org/10.5489/cuaj.6186

Published online November 29, 2019

$* * *$

\section{Introduction}

Brunn's cysts originate from Von Brunn's nests, which are considered a normal variation of urothelium formed by the sequestering of epithelial nests from urothelial buds. ${ }^{1}$ Von Brunn's nests are not visible endoscopically, but may be present in up to $90 \%$ of bladders and consist histologically of solid nests of benign urothelial cells found within the lamina propria or submucosa at the trigone. ${ }^{1}$ Bladder outlet obstruction caused by Brunn's cysts is rare, with limited reported cases in the literature, all of which are described in males. ${ }^{2-4}$

\section{Case report}

A previously healthy 37-year-old male was referred with new onset obstructive voiding symptoms. He had been complaining of weak stream, hesitancy, straining, and incomplete emptying for 2 months, with an IPSS score of 10 and bother score of 5 . He denied gross hematuria, dysuria, nocturia, urinary incontinence, urinary tract infections, or any history of smoking or bladder irritants. Urinalysis, urine culture, and renal function studies were normal. Ultrasound of the kidneys, ureter, and bladder showed a $1.7 \mathrm{~cm}$ thin-walled midline cystic structure in the bladder neck, abutting the ureteric orifice (Fig 1) without hydronephrosis. He had an elevated post-void residual (PVR) volume of $110 \mathrm{ml}$.

Cystoscopic examination demonstrated a spherical mass at the 12 o'clock position anteriorly on the bladder neck, seen best on retroflexion (Fig 2A). The mass can be seen causing a ball-valve effect at the bladder neck (Fig 2B). The rest of bladder was found to be unremarkable, with normal urothelium and ureteric orifices. The mass was separate from the prostate, which was small and non-obstructing (Fig 2C). The patient was taken to the operating room for a transurethral resection of the bladder neck lesion (Fig 2D). The mass deflated upon resection and there was no evidence of any solid components. At follow-up one month after surgery, the patient's obstructive voiding symptoms had completely resolved and his PVR was 0 
$\mathrm{ml}$. The patient's repeat IPSS score was 2 with a bother score of 1 . Final pathology report was consistent with a Brunn's cyst with no evidence of dysplasia or malignancy.

The differential diagnosis of other cystic lesions near the bladder neck should always be considered. Cystitis cystica - reactive chronic inflammation secondary to bladder irritants typically leads to small filling defects within the bladder wall, can also manifest as a large cystic lesion. ${ }^{5}$ In these patients, inflammatory cells are commonly seen on urine cytology and the bladder mucosa has an inflammatory appearance. ${ }^{6}$ Cystitis glandularis is also associated with chronic inflammation leading to glandular metaplasia. Cystitis glandularis can also cause hematuria and has been reported to cause acute urinary retention. ${ }^{7}$ Infravesical cystic lesions in the prostate are rare, but have been reported to be associated with lower urinary tract symptoms as well. ${ }^{8}$ Most prostatic cystic lesions are found incidentally on imaging and can been classified as medial cysts, cysts of the ejaculatory duct, parenchymal cysts, infectious cysts, cystic tumours, or cysts secondary to parasitic disease. ${ }^{9}$ Ureteroceles from ectopic ureteric insertions can also appear as cystic structures near the bladder neck. However, the majority are congenital, associated with duplex collecting systems, and present in childhood. ${ }^{10}$

\section{Conclusions}

Brunn's cyst is a rare cause of bladder outlet obstruction and can be considered in young patients with new onset of obstructive lower urinary tract symptoms. First-line treatment has been universally described as transurethral deroofing and resection, which results in durable resolution of voiding symptoms with no reported recurrences. ${ }^{2-4}$ Given the benign nature of the pathology, routine surveillance in the absence of symptoms appears to be unnecessary.

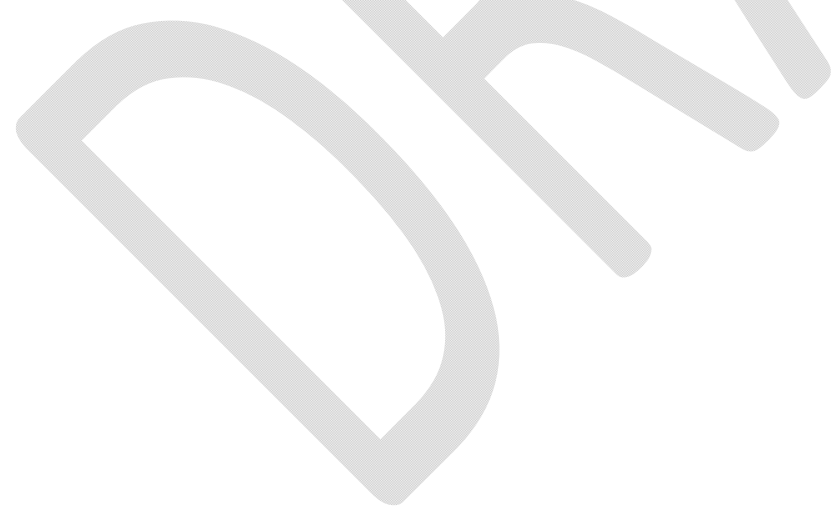




\section{References}

1. Wiener DP., Koss LG., Sablay B., et al. The prevalence and significance of Brunn's nests, cystitis cystica and squamous metaplasia in normal bladders. J Urol 1979;122(3):317-21. Doi: 10.1016/s0022-5347(17)56384-3.

2. Franco I., Eshghi M., Schutte H., et al. Bladder neck obstruction secondary to Brunn's cyst. J Urol 1988;139(1):126-7. Doi: 10.1016/s0022-5347(17)42316-0.

3. Grimsby GM., Tyson MD., Salevitz B., et al. Bladder outlet obstruction secondary to a Brunn's cyst. Curr Urol 2012;6(1):50-2. Doi: 10.1159/000338871.

4. Ilyas M., Shafi F., Choh N. Brunn's cyst: A rare cause of lower urinary tract symptoms. Indian J Urol 2018;34(3):231-2. Doi: 10.4103/iju.IJU_117_18.

5. Potts S., Calleary J. Cystitis Cystica as a Large Solitary Blad̄er Cyst. J Endourol Case Reports 2017;3(1):34-8. Doi: 10.1089/cren.2017.0010.

6. Bastianpillai C., Warner R., Beltran L., et al. Cystitis cystica and glandularis producing large bladder masses in a 16-year-old boy. JRSM Open 2018;9(3):20542704177460602054270417746060. Doi: 10.1177/2054270417746060.

7. Michajłowski J., Matuszewski M., Kłącz J., et al. Acute urinary retention in a patient with extended cystitis glandularis. Cent Eur J Urol 2011;64(2):94-6. Doi: 10.5173/ceju.2011.02.art11.

8. Saha B., Sinha RK., Mukherjee S., et al. Midline prostatic cyst in a young man with lower urinary tract symptoms. BMJ Case Rep 2014;2014:1-2. Doi: 10.1136/bcr-2014207816.

9. Galosi AB., Montironi R., Fabiani A., et al. Cystic lesions of the prostate gland: an ultrasound classification with pathological correlation. J Urol 2009;181(2):647-57. Doi: 10.1016/j.juro.2008.10.006.

10. Jain S., Chen F. Developmental pathology of congenital kidney and urinary tract anomalies. Clin Kidney J 2018;12(3):382-99. Doi: 10.1093/ckj/sfy112. 


\section{Figures and Tables}

Fig. 1. Bladder ultrasound depicting cystic mass at anterior bladder neck.

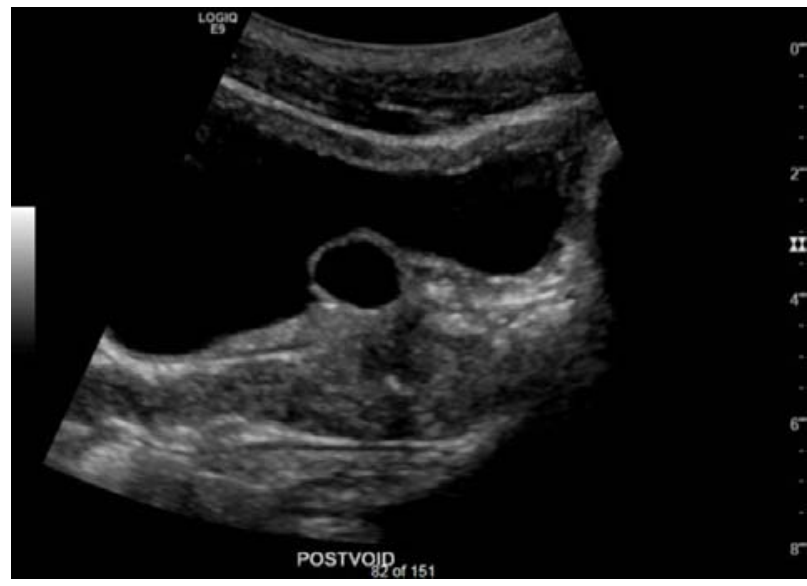

Fig. 2. Cystoscopic evaluation of patient. (A) Brunn's cyst seen on retroflexion;

(B) anterior cyst seen at bladder neck; (C) verumontanum and normal prostatic urethra; (D) retroflexion view post-transurethral resection.

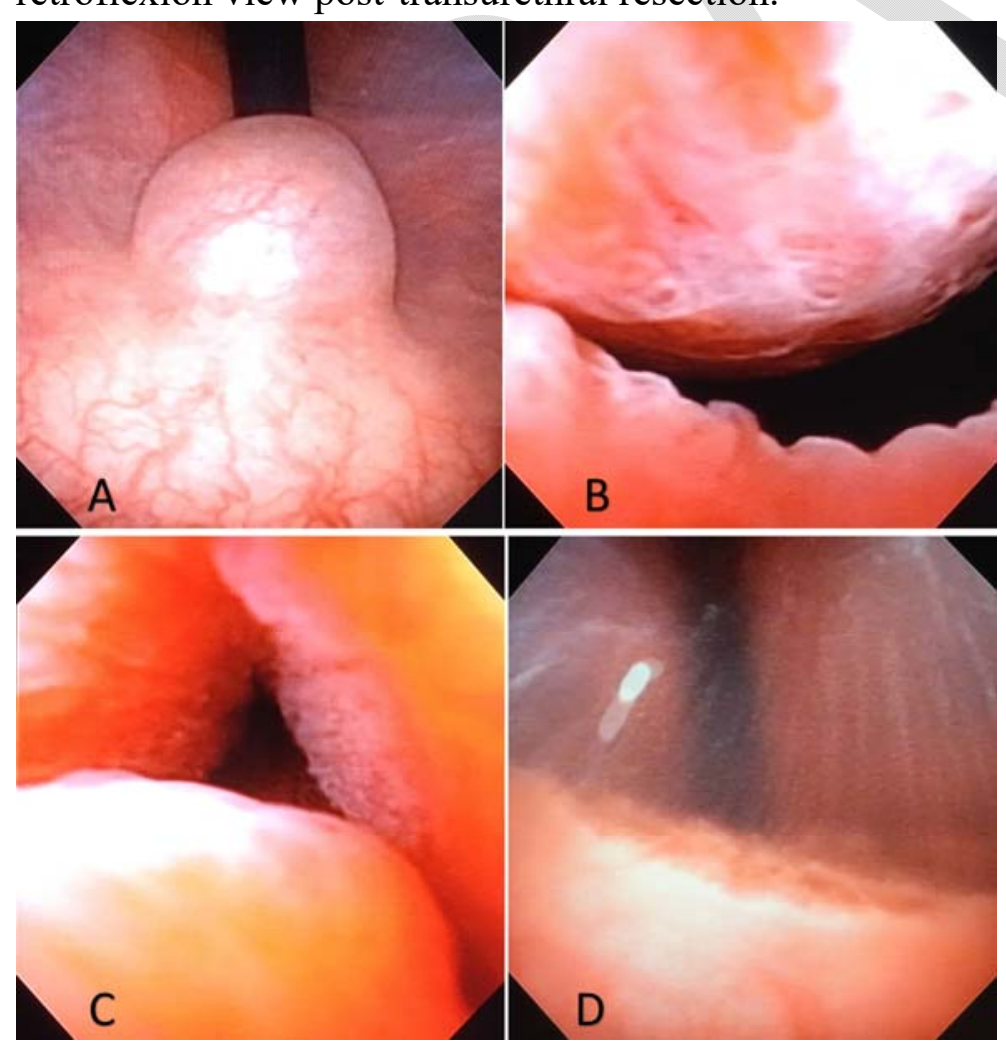

